\title{
Investigation of optimal sample preparation conditions with potassium triiodide and optimal imaging settings for microfocus computed tomography of excised cat hearts
}

\author{
Ian C. Simcock MS \\ J. Ciaran Hutchinson MB, MS \\ Susan C. Shelmerdine MB, MS \\ Jose Novos Matos DVM \\ Neil J. Sebire MD \\ Virginia Luis Fuentes $\mathrm{PhD}$ \\ Owen J. Arthurs PhD
}

Received March 15, 2019

Accepted August 7, 2019.

From the Departments of Radiology (Simcock, Shelmerdine, Arthurs) and Pathology (Hutchinson, Sebire), Great Ormond Street Hospital for Children, National Health Service Foundation Trust, London WCIN 3JH, England; National Institute for Health Research, Great Ormond Street Hospital, Biomedical Research Centre, University College London, Great Ormond Street Institute of Child Health, Research and Development Office, London WCIN IEH, England (Simcock, Hutchinson, Shelmerdine, Arthurs); and Department of Clinical Sciences, Royal Veterinary College, University of London, London NWI OTU, England (Matos, Luis Fuentes). Dr. Matos' present address is the Clinical Department, Department of Veterinary Medicine, University of Cambridge, Cambridge CB3 OES, England.

Address correspondence to Mr. Simcock (ian.simcock@gosh.nhs.uk).

\begin{abstract}
OBJECTIVE
To determine optimal sample preparation conditions with potassium triiodide $\left(\mathrm{I}_{2} \mathrm{KI}\right)$ and optimal imaging settings for microfocus CT (micro-CT) of excised cat hearts.
\end{abstract}

\section{SAMPLE}

7 excised hearts (weight range, 10 to $17.6 \mathrm{~g}$ ) obtained from healthy adult cats after euthanasia by IV injection of pentobarbital sodium.

\section{PROCEDURES}

Following excision, the hearts were preserved in $10 \%$ formaldehyde solution. Six hearts were immersed in $1.25 \% \mathrm{I}_{2} \mathrm{KI}$ solution $(n=3)$ or $2.5 \% \mathrm{I}_{2} \mathrm{KI}$ solution (3) for a 12-day period. Micro-CT images were acquired at time 0 (prior to iodination) then approximately every 24 and 48 hours thereafter to determine optimal sample preparation conditions (ie, immersion time and concentration of $\mathrm{I}_{2} \mathrm{KI}$ solution). Identified optimal conditions were then used to prepare the seventh heart for imaging; changes in voltage, current, exposure time, and gain on image quality were evaluated to determine optimal settings (ie, maximal signal-to-noise and contrast-to-noise ratios). Images were obtained at a voxel resolution of $30 \mu \mathrm{m}$. A detailed morphological assessment of the main cardiac structures of the seventh heart was then performed.

\section{RESULTS}

Immersion in $2.5 \% \mathrm{I}_{2} \mathrm{KI}$ solution for 48 hours was optimal for sample preparation. The optimal imaging conditions included a tube voltage of $100 \mathrm{kV}$, current of $150 \mu \mathrm{A}$, and exposure time of 354 milliseconds; scan duration was 12 minutes.

\section{CONCLUSIONS AND CLINICAL RELEVANCE}

Results provided an optimal micro-CT imaging protocol for excised cat hearts prepared with $\mathrm{I}_{2} \mathrm{KI}$ solution that could serve as a basis for future studies of micro-CT for high resolution 3-D imaging of cat hearts. (Am J Vet Res 2020;81:326-333)
M icrofocus CT allows 3-D imaging of specimens with a voxel resolution of $0.001 \mathrm{~mm}^{1-8}$ This technique has been used in imaging studies of bone and teeth, ${ }^{9-11}$ but also could potentially be used to evaluate and differentiate soft tissue structures (eg, myocardium) with the use of contrast material. ${ }^{12-18}$ The ability to obtain detailed images by micro-CT could prove useful for morphological studies of diseases such as hypertrophic cardiomyopathy, a condition that is estimated to affect $15 \%$ of cats ${ }^{19}$ and that has a wide range of gross anatomic and histopathologic features. ${ }^{20}$ To date, morphological studies of cats with hypertrophic cardiomyopathy have

$\begin{array}{ll}\text { ABBREVIATIONS } \\ \text { CNR } & \text { Contrast-to-noise ratio } \\ \mathrm{I}_{2} \mathrm{KI} & \text { Potassium triiodide } \\ \text { Micro-CT } & \text { Microfocus CT } \\ \text { ROI } & \text { Region of interest } \\ \text { SNR } & \text { Signal-to-noise ratio }\end{array}$

mostly focused on qualitative features, and histopathologic evaluation is limited by sampling methods. ${ }^{21}$

Some micro-CT studies have been conducted on hearts from human fetuses, ${ }^{22}$ mice, ${ }^{23}$ and rabbits. ${ }^{24}$ However, to the authors' knowledge, the optimal sample processing and imaging protocol for micro-CT of cardiac structures in cats has not been previously evaluated. Although various contrast agents have been evaluated for micro-CT of soft tissues, ${ }^{25}$ osmium tetroxide, phosphotungstic acid, and $\mathrm{I}_{2} \mathrm{KI}$ solutions have been used most extensively. Osmium tetroxide provides excellent soft tissue contrast but is highly toxic and expensive. ${ }^{26}$ Phosphotungstic acid is more affordable but can take up to 12 days to completely stain large specimens, ${ }^{27}$ which can result in distortion of histologic features ${ }^{28}$; it is unsuitable for staining samples that have a volume $>1 \mathrm{~cm}^{3} .{ }^{4}$ Potassium triiodide is widely available, stable, and affordable; in addition, it has low toxicity, can diffuse through 
soft tissue samples that are up to a few centimeters in thickness, and can be removed after imaging. 2,15,23,29 Large specimens (ie, human fetuses) have been successfully imaged by micro-CT after preparation with $\mathrm{I}_{2} \mathrm{KI}$ solution. ${ }^{22,30}$ In a micro-CT study ${ }^{7}$ of the neuroanatomy of rat brains, methods for optimization of sample preparation (in vivo and ex vivo specimens) were reported. However, other micro-CT imaging studies $^{2,22-24,31,32}$ in which $\mathrm{I}_{2} \mathrm{KI}$ solution was used for sample preparation, most of which cite the methods of Degenhardt et al, ${ }^{14}$ have used a wide range of iodine concentrations and imaging settings and lacked evaluation of optimal conditions for sample preparation. In addition to optimal conditions for sample preparation, optimal image acquisition settings are necessary to obtain high-quality micro-CT images with maximal contrast and signal intensity. ${ }^{16,33}$

With appropriate optimization, micro-CT could potentially be useful for evaluation of cardiac structures in cats, including chamber volume quantification, analysis of papillary muscle geometry and mitral valve length, and 3-D distribution of myofiber disarray. The objective of the study reported here was to determine optimal sample preparation conditions (ie, immersion time and concentration of $\mathrm{I}_{2} \mathrm{KI}$ solution) and optimal imaging settings (ie, voltage, current, exposure time, and gain) for micro-CT of excised hearts from healthy adult cats.

\section{Materials and Methods}

\section{Sample}

Seven hearts excised from healthy adult cats were sourced as a convenience sample from the control arm of a study of feline cardiomyopathy (approved by the Clinical Research Ethical Review Board of the Royal Veterinary College [URN 2016 1638-3]) that followed Directive 2010/63/EU of the European Union on the protection of animals used for scientific purposes. The hearts ranged in weight from 10 to $17.6 \mathrm{~g}$. Cats were euthanized by IV injection of pentobarbital sodium for reasons unrelated to this study. For the first part of this 2-part study, 6 hearts (from 3 female and 3 male cats; age range, 6 to 14 years) were used to determine optimal sample preparation conditions. For the second part, the seventh heart (from a 5-year-old male cat) was prepared by use of the identified optimal conditions and underwent evaluation to determine the optimal micro-CT imaging settings.

\section{Sample preparation}

Immediately after euthanasia of the cats, each heart was excised and flushed with saline $(0.9 \% \mathrm{NaCl})$ solution, then preserved in $10 \%$ formaldehyde solution. Prior to immersion in $\mathrm{I}_{2} \mathrm{KI}$ solution, the hearts were washed in water, towel dried, and weighed. Hearts were immersed in $1.25 \% \mathrm{I}_{2} \mathrm{KI}$ ( $\mathrm{n}=3$; weight range, 10.0 to $15.0 \mathrm{~g}$ ) or $2.5 \% \mathrm{I}_{2} \mathrm{KI}$ (3; weight range, 10.8 to $16.8 \mathrm{~g}$ ) solution; these concentrations of $\mathrm{I}_{2} \mathrm{KI}$ solution were selected on the basis of a literature review (Appendix). A 5\% $\mathrm{I}_{2} \mathrm{KI}$ solution (100 $\mathrm{g}$ of potassium iodide and $50 \mathrm{~g}$ of $\mathrm{I}_{2}$ in $1 \mathrm{~L}$ of water) was diluted by adding an equal volume of $10 \%$ formalin solution to create a $2.5 \% \mathrm{I}_{2} \mathrm{KI}$ solution; a portion of the $2.5 \% \mathrm{I}_{2} \mathrm{KI}$ solution was similarly diluted with an equal volume of $10 \%$ formalin solution to create a $1.25 \% \mathrm{I}_{2} \mathrm{KI}$ solution. Each of the 6 hearts was immersed in the assigned $\mathrm{I}_{2} \mathrm{KI}$ solution for a 12-day period at room temperature (approx $20^{\circ} \mathrm{C}$ ); to ensure diffusion of $\mathrm{I}_{2} \mathrm{KI}$ solution under constant hydrostatic pressure, the volume of solution in which each heart was immersed was determined as 30 multiplied by the heart weight (with grams converted to milliliters).

To determine optimal sample preparation conditions (ie, concentration of $\mathrm{I}_{2} \mathrm{KI}$ solution and immersion time), hearts underwent serial imaging starting at 0 hours (prior to iodination) and then approximately every 24 and 48 hours thereafter over the 12-day period until it was apparent that maximal iodination was achieved by use of a micro-CT scan-

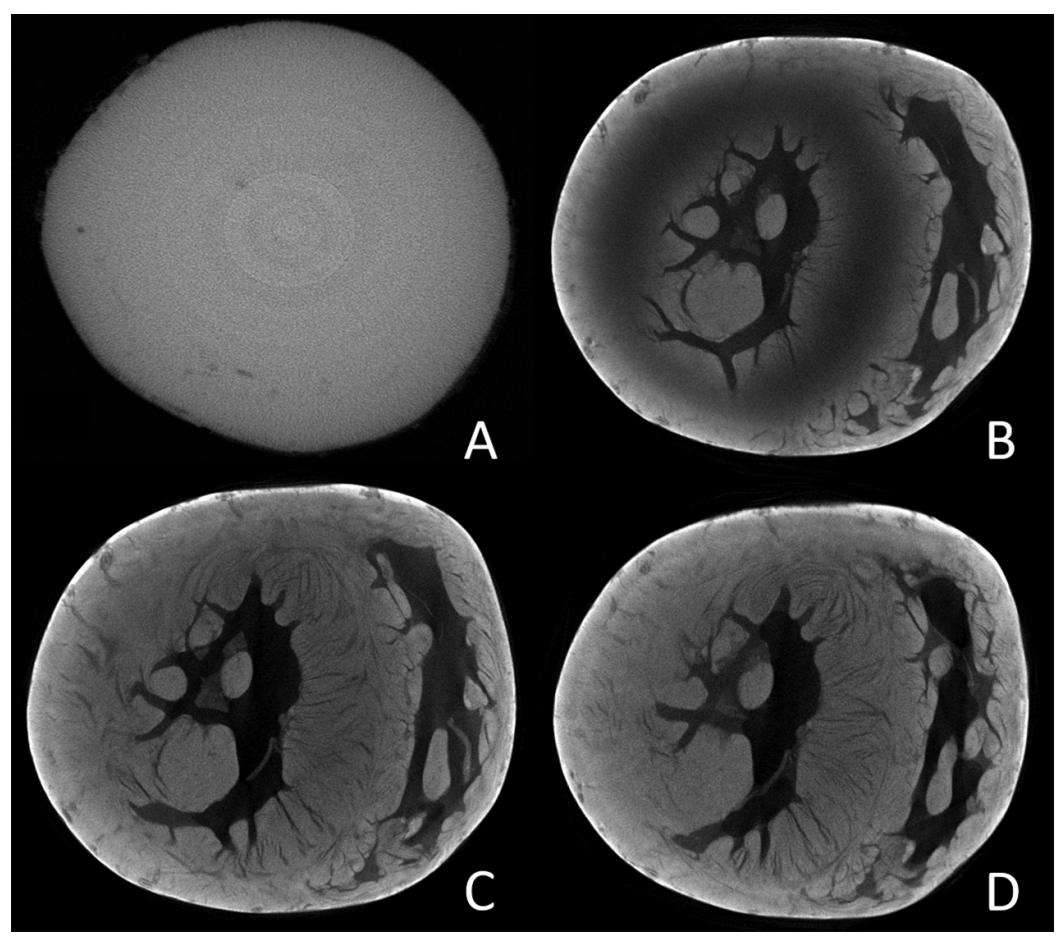

Figure I-Short-axis micro-CT images obtained at the level of the left ventricular papillary muscle of a representative healthy adult cat heart that was excised and preserved in $10 \%$ formaldehyde solution, then washed in water and dried prior to immersion in $2.5 \% \mathrm{I}_{2} \mathrm{KI}$ solution. A-Prior to iodination ( 0 hour), no internal structure is seen. B-After 24 hours of immersion, iodination is improved, although a halo of darkness around the left ventricle is still present. C-After 48 hours of immersion, maximal iodination of myocardial muscle fibers is achieved. $D$-After 72 hours of immersion, the degree of iodination differs little from that detected after 48 hours of immersion. 
ner $^{\mathrm{a}}$ with a tungsten target. Prior to imaging, hearts were drained, rinsed, towel dried, and wrapped in plastic film ${ }^{\mathrm{b}}$ to prevent dehydration during the scanning process. The 6 hearts underwent imaging with a constant resolution of $30 \mu \mathrm{m}$, tube voltage of $100 \mathrm{kV}$, current of $150 \mu \mathrm{A}$, power of $15 \mathrm{~W}$, exposure time of 354 milliseconds, gain of $24 \mathrm{~dB}, 1$ frame/projection, and 2,712 projections $/ 360^{\circ}$ rotation of the heart (optimized number of projections determined on the basis of the size of the specimen and the distance from the detectors); scan duration was 16 minutes.

\section{Imaging settings}

After the optimal sample preparation conditions were determined for the 6 hearts in the first part of the study, the identified conditions were used to prepare the seventh heart (weight, $17.6 \mathrm{~g}$ ). This heart was then used in the second part of the study to evaluate the effect of alterations in imaging settings (ie, voltage, current, exposure time, and gain) on image quality. The range for each setting was chosen to allow optimal contrast of soft tissue within the limitations of the scanner. Prior to imaging, the heart was drained, rinsed, towel dried, and wrapped in plastic film to prevent dehydration during the scanning process. The same micro-CT scanner ${ }^{\mathrm{a}}$ with a tungsten target as that used for determination of optimal sample preparation conditions was used for determination of optimal imaging settings. For evaluation of each imaging setting (voltage, current, exposure time, and gain), all settings were kept constant apart from the setting under evaluation. Other conditions (ie, a constant resolution of $30 \mu \mathrm{m}$, 1 frame/projection, and 2,712 projections $/ 360^{\circ}$ rotation of the heart) were kept constant throughout this part of the study. The identified optimal settings were used to perform a detailed morphological assessment of the main cardiac structures of the seventh heart through reconstructed 3-D images.

\section{Calculation of SNRs and CNRs and statistical analysis}

For each heart, signal intensity measurements within 9 ROIs ( 3 in the inner portion of the myocardium, 3 in the outer portion of the myocardium, and 3 in the air surrounding heart) that had been subjectively placed by a single interpreter were obtained. These measurements were used to calculate the mean \pm SD signal intensity values and to determine SNRs and CNRs. The SNR was defined as the mean signal intensity for each portion of the myocardium ROIs divided by the SD of the signal intensity values. The CNR was defined as the mean difference in signal intensity measurements for each myocardial ROI and its corresponding air ROI divided by the SD of the signal intensity values for air. The Shapiro-Wilk test was used to assess the data distributions for normality. For each heart, a Student $t$ test was used to compare mean SNRs at various time points; mean CNRs were similarly compared. A paired $t$ test was used for repeated measures to determine the earliest time at which a plateau in signal intensity measurements was achieved (ie, no significant increase in signal intensity detected after the plateau was achieved). Statistical analyses were performed with statistical software. ${ }^{c}$ Significance was defined as a value of $P<0.05$.

\section{Results}

\section{Sample preparation}

Immersion in $1.25 \%$ or $2.5 \% \mathrm{I}_{2} \mathrm{KI}$ solution resulted in gradual iodination of all 6 hearts, with maximum iodination achieved after 48 to 100 hours of immersion; no appreciable increase in signal intensity was noted after 72 hours for 4 hearts (Figure I). A plateau in signal intensity values (ie, no further significant increase in signal intensity) was achieved at a faster rate for the outer (vs inner) portion of the myocardium and in $2.5 \%$ (vs $1.25 \%$ ) $\mathrm{I}_{2} \mathrm{KI}$ solution (Figure 2). The earliest time to a plateau in signal intensity values was summarized by concentration of $\mathrm{I}_{2} \mathrm{KI}$ solution used and location in the myocardium (Table I). After the identified start of the signal intensity plateau period for each myocardial region exposed to each concentration of $\mathrm{I}_{2} \mathrm{KI}$ solution, the percentage change in SNR or CNR was $<5 \%$. The differences in SNRs between the outer and inner portions of the myocardium after immersion in $1.25 \%$ or $2.5 \% \mathrm{I}_{2} \mathrm{KI}$ solution plateaued at approximately the same times when the plateaus for signal intensity commenced. On the basis of the findings for this part of the study, we selected immer-

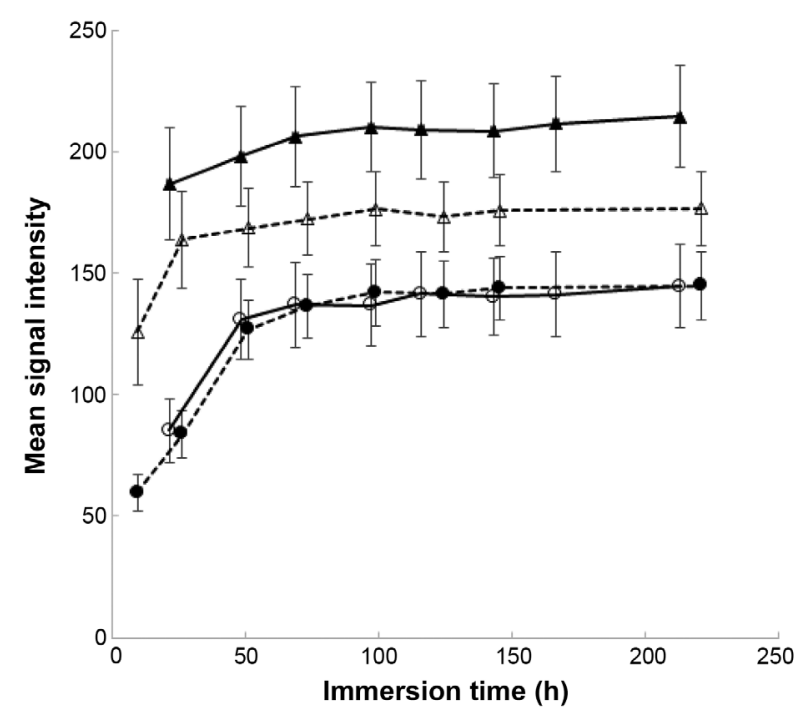

Figure 2-Mean \pm SE signal intensity values obtained by micro-CT imaging of hearts from 6 healthy adult cats that were excised, preserved in $10 \%$ formalin solution, then washed in water and dried prior to immersion in $1.25 \%(n=3$; dashed lines) or $2.5 \%$ (3; solid lines) $\mathrm{I}_{2} \mathrm{KI}$ solution. Images of each heart were obtained prior to iodination ( 0 hours) and every 24 to 48 hours thereafter (until maximal iodination was achieved); signal intensity measurements were obtained for 3 ROls in the inner portion of the myocardium (circles) and 3 ROls in the outer portion of the myocardium (triangles), and an overall mean value for each portion of the myocardium in the 6 hearts was calculated for each time point. 
Table I-Earliest time to plateau in signal intensity and percentage signal intensity change thereafter by myocardial location in hearts excised from 6 healthy adult cats and immersed in $1.25 \%(n=3)$ or $2.5 \%(3) \mathrm{I}_{2} \mathrm{KI}$ solution, as determined by imaging with a micro-CT scanner prior to iodination ( 0 hours) and approximately every 24 to 48 hours thereafter over a 9-day period.

\begin{tabular}{llcc}
$\begin{array}{c}\text { Concentration } \\
\text { of } \mathrm{I}_{\mathbf{2}} \mathrm{KI} \text { solution }\end{array}$ & Location in myocardium & $\begin{array}{c}\text { Range of immersion } \\
\text { time to plateau }(\mathbf{h})\end{array}$ & $\begin{array}{c}\text { Range of percentage } \\
\text { change after start of plateau }\end{array}$ \\
\hline $2.5 \%$ & Outer & $21-48$ & $<4.18$ \\
& Inner & $48-68$ & $<3.39$ \\
$1.25 \%$ & Difference* (outer - inner) & $21-48$ & - \\
& Outer & $73-99$ & $<3.48$ \\
& Inner & $50-73$ & $<2.80$ \\
& Difference* (outer - inner) & $25-50$ & - \\
\hline
\end{tabular}

For each heart, signal intensity measurements within 9 ROIs ( 3 in the inner portion of the myocardium, 3 in the outer portion of the myocardium, and 3 in the air surrounding the heart) that had been subjectively placed by a single interpreter were obtained. The SNR was defined as the mean signal intensity for each portion of the myocardium and air ROls divided by the SD of the signal intensity values. The overall mean SNR for all 6 hearts at each time point was calculated. A Student $t$ test was used to compare mean SNRs at various time points. For each heart, a paired $t$ test for repeated measures was used to determine the earliest time to signal intensity plateau (ie, no significant increase in signal intensity after the plateau was achieved).

*Values represent the range of differences in SNR values between the outer and inner portions of the myocardium.

$-=$ Not applicable.
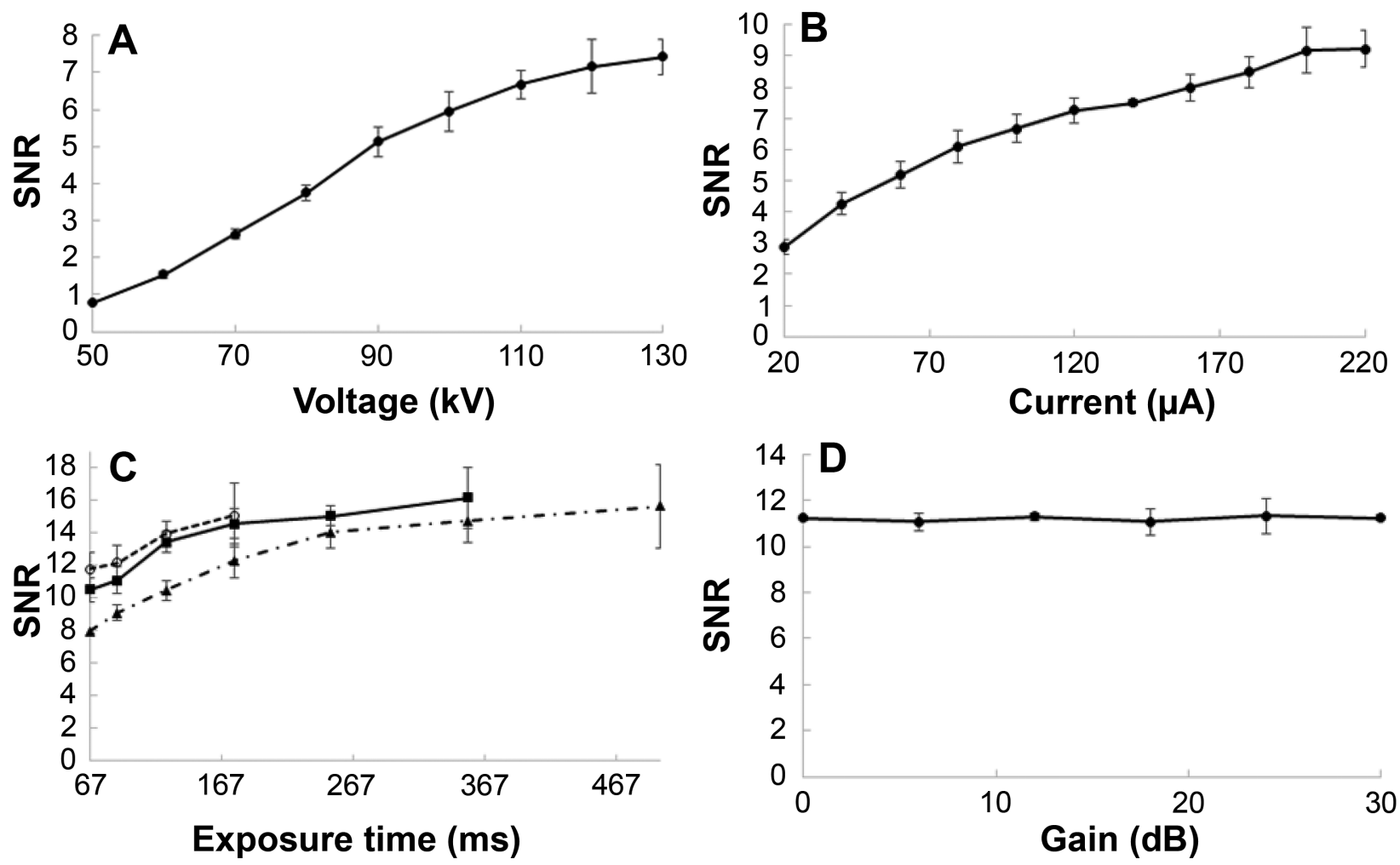

Figure 3-Mean \pm SE SNRs obtained by micro-CT imaging of a healthy 5-year-old cat's heart that was prepared by use of optimal sample preparation conditions (ie, immersion in $2.5 \% \mathrm{I}_{2} \mathrm{Kl}$ solution for 48 hours) that were determined by evaluation of 6 cat hearts. At a resolution of $30 \mu \mathrm{m}$, images were obtained as the tube voltage was increased with a constant current of I30 $\mu \mathrm{A}$, exposure time of 250 milliseconds, and gain of $24 \mathrm{~dB}(\mathrm{~A})$; as the current was increased with a constant tube voltage of $100 \mathrm{kV}$, exposure time of 250 milliseconds, and gain of $24 \mathrm{~dB}(\mathrm{~B})$; as the exposure time was increased with a constant tube voltage of $100 \mathrm{kV}$, gain of 24 $\mathrm{dB}$, and currents of $100 \mu \mathrm{A}$ (triangles), $150 \mu \mathrm{A}$ (squares), and $200 \mu \mathrm{A}$ (circles; $\mathrm{C}$ ); and as gain was increased with a constant tube voltage of $100 \mathrm{kV}$, current of $150 \mu \mathrm{A}$, and exposure time of 354 milliseconds (D). See Figure 2 for remainder of key.

sion in $2.5 \% \mathrm{I}_{2} \mathrm{KI}$ solution for 48 hours as optimal for preparation of the seventh heart to determine of optimal imaging settings.

\section{Imaging settings}

For the seventh heart that was prepared by use of optimal sample preparation conditions, the SNR and CNR increased in a linear manner as the tube voltage increased from 50 to $130 \mathrm{kV}$ with a constant current of
$130 \mu \mathrm{A}$, exposure time of 250 milliseconds, and gain of $24 \mathrm{~dB}$ (scan duration of 13 minutes; Figures 3 and 4). The increases in SNR and CNR were not significant at tube voltages $>110$ and $100 \mathrm{kV}$, respectively. Accordingly, a tube voltage of $100 \mathrm{kV}$ was used for all subsequent evaluations of the effects of alterations in settings for current, exposure time, and gain.

For the seventh heart, the SNR and CNR increased in a linear manner as the current increased 

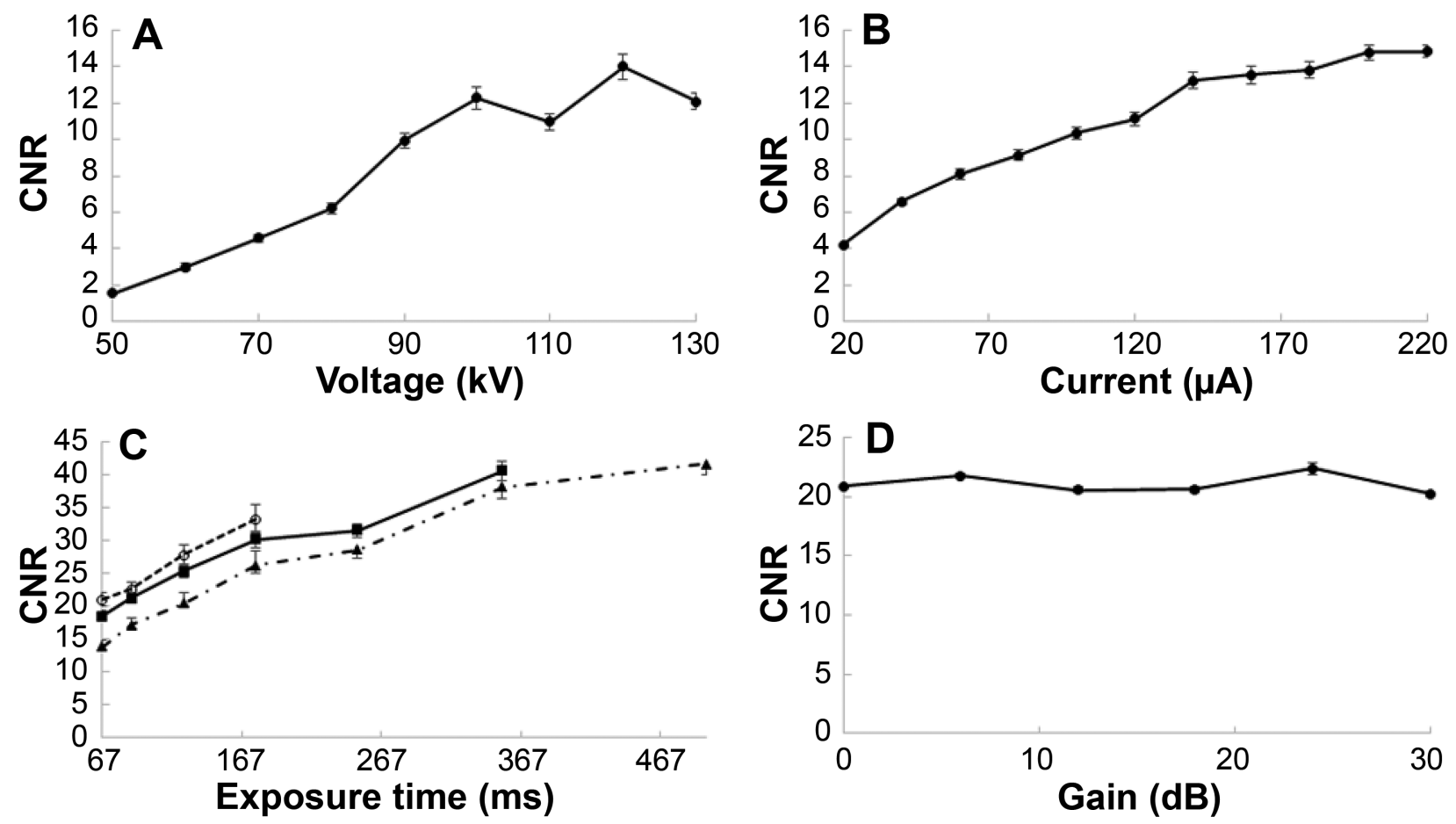

Figure 4-Mean \pm SE CNRs obtained during micro-CT imaging of the cat heart represented in Figure 3 . See Figures 2 and 3 for remainder of key.

from 20 to $220 \mu \mathrm{A}$ with a constant tube voltage of $100 \mathrm{kV}$, exposure time of 250 milliseconds, and gain of $24 \mathrm{~dB}$; the maximal SNR and CNR were observed at a current of $220 \mu \mathrm{A}$ (Figures 3 and 4). When evaluated separately at currents of $100 \mu \mathrm{A}$, $150 \mu \mathrm{A}$, and $200 \mu \mathrm{A}$, the SNR and CNR increased as the exposure time increased from 67 to 500 milliseconds with a constant tube voltage of $100 \mathrm{kV}$ and gain of $24 \mathrm{~dB}$. Maximal SNR and CNR during evaluation of changes in exposure time were observed at currents of $100 \mu \mathrm{A}$ (15.63 and 41.62, respectively, at an exposure time of 500 milliseconds) and 150 $\mu \mathrm{A}$ (16.16 and 40.54, respectively, at an exposure time of 354 milliseconds). Because the scan duration with a current of $150 \mu \mathrm{A}$ was shorter than that with a current of $100 \mu \mathrm{A}$ (12 vs 17 minutes), a current of $150 \mu \mathrm{A}$ and an exposure time of 354 milliseconds were selected as optimal settings. As gain increased from 0 to $30 \mathrm{~dB}$ with a constant tube voltage of $100 \mathrm{kV}$, current of $150 \mu \mathrm{A}$, and exposure time of 354 milliseconds, no significant change in SNR or CNR was noted. On the basis of the findings for this part of the study, settings considered optimal for micro-CT of the seventh cat heart were as follows: tube voltage of $100 \mathrm{kV}$, current of $150 \mu \mathrm{A}$, and exposure time of 354 milliseconds.

\section{Imaging results}

Use of a micro-CT protocol that included the identified optimal sample preparation conditions and optimal imaging settings allowed clear identification of valves, atrial appendages, papillary mus- cles, ventricular trabeculae, and myocardial structure of the seventh cat heart (Figure 5). Fat deposits in the epicardial and intramyocardial portions of the heart were readily identified. In addition, the coronary arteries could be seen from their origin at the sinus of Valsalva to their smaller intramyocardial branches.

\section{Discussion}

In the present study, a protocol that included optimal sample preparation conditions and optimal imaging settings for micro-CT of excised hearts from healthy adult cats was devised. Immersion of hearts in $2.5 \% \mathrm{I}_{2} \mathrm{KI}$ solution for 48 hours and imaging settings of $100 \mathrm{kV}$ for tube voltage, $150 \mu \mathrm{A}$ for current, and 354 milliseconds for exposure time were optimal for micro-CT of the hearts included in this study. Although optimal sample preparation conditions and imaging settings may differ among species and organs, these findings could serve as a useful starting point for future studies involving micro-CT of hearts of various sizes. The types of reconstructed 3-D images obtained in the present study would be useful for chamber volume quantification and complex morphological assessment for cardiomyopathies and congenital heart defects.

Compared with conditions used in other studies (Appendix), the method for sample preparation established in the present study resulted in full iodination of the excised hearts by use of a low concentration of $\mathrm{I}_{2} \mathrm{KI}$ solution $(2.5 \%$ vs $3.75 \%$ or $7.5 \%)$ and a short immersion time (2 days vs 5 and 7 


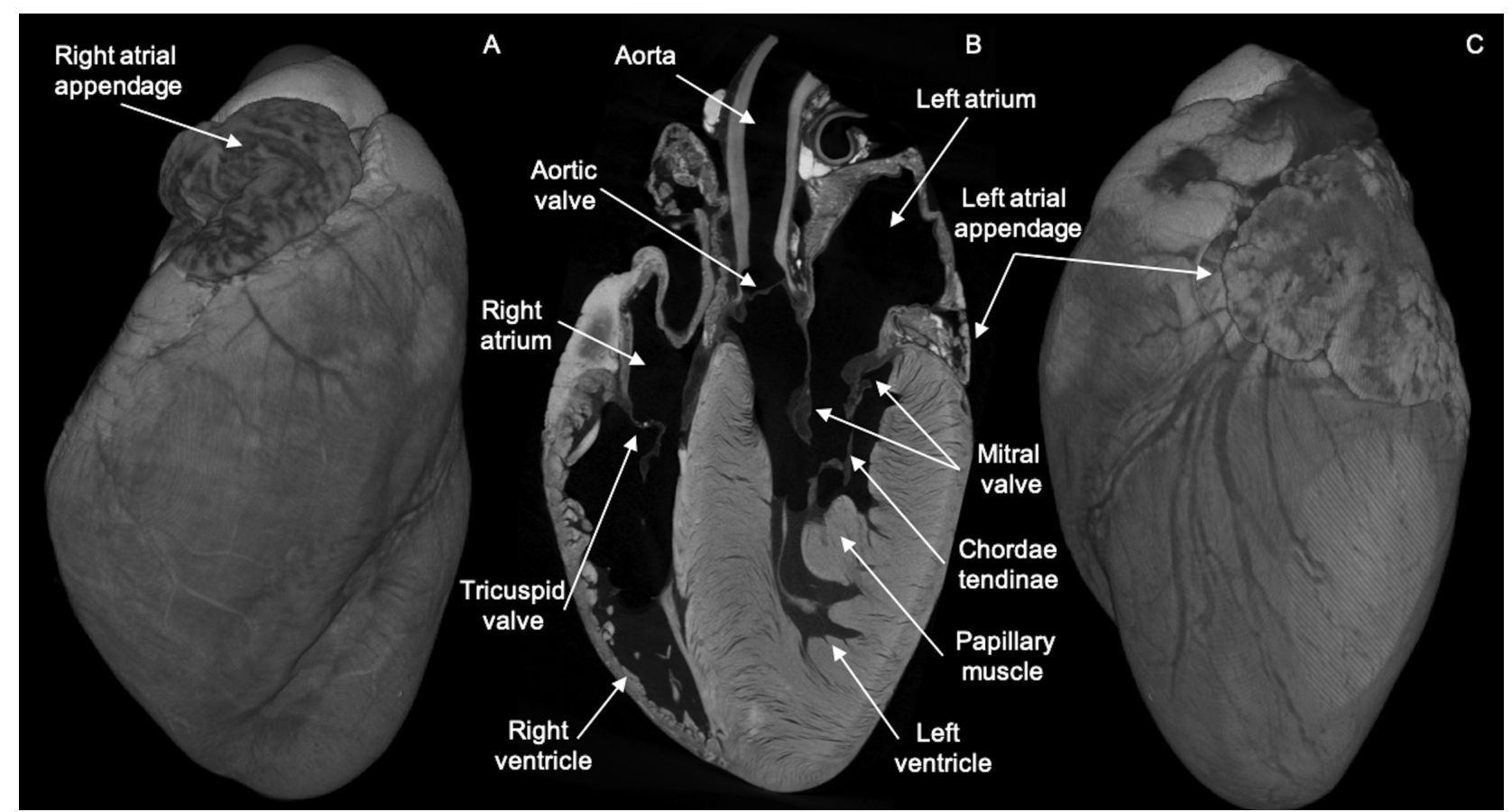

Figure 5-Reconstructed micro-CT 3-D volume rendered images (obtained at a resolution of $30 \mu \mathrm{m})$ of the right lateral (A) and ventrocranial $(C)$ external aspects and 5 -chamber long-axis view (B) of the cat heart represented in Figure 3 . Images were obtained with the optimal micro-CT protocol involving immersion of the heart in $2.5 \% \mathrm{I}_{2} \mathrm{KI}$ solution for 48 hours followed by imaging with tube voltage of $100 \mathrm{kV}$, current of I50 $\mathrm{AA}$, and exposure time of 354 milliseconds. See Figures 2 and 3 for remainder of key.

days in studies of excised rabbit hearts ${ }^{24}$ and a dog heart, ${ }^{5}$ respectively). Other more extreme reported conditions for preparation of soft tissue samples for micro-CT include use of $\mathrm{I}_{2} \mathrm{KI}$ solutions of $0.3 \%{ }^{34}$ to $25 \%{ }^{35}$ and immersion times of 30 minutes $^{12}$ to 7 weeks, ${ }^{35}$ depending on the size and type of sample. The use of various methods by different authors is not surprising, given that clinical use of micro-CT scanners is not widespread. By optimizing imaging settings to maximize SNR and CNR in the present study, we obtained detailed images for evaluation of cardiac structures, yet minimized the possibility of morphological distortion of those cardiac structures that could result from prolonged exposure to high concentrations of $\mathrm{I}_{2} \mathrm{KI}$ solution. ${ }^{14,22,31,36}$

The use of $\mathrm{I}_{2} \mathrm{KI}$ solution as a micro-CT soft tissue contrast agent is dependent on the rapid diffusion of the iodine molecules into the tissue, where they bind to glycogen within the muscle cells. ${ }^{12,24}$ Because of the high atomic number of iodine, differential photon attenuation provides excellent tissue contrast. ${ }^{37}$ However, the precise concentration of $\mathrm{I}_{2} \mathrm{KI}$ solutions applied by other researchers is difficult to determine because of use of the term Lugol solution, which historically refers to the fact that a certain weight of $\mathrm{I}_{2}$ with double the weight of KI has been dissolved in water without stating the concentration of the individual components. Some sources refer to 5\% Lugol solution as being normal or full strength, ${ }^{29}$ but these latter terms are not consistently applied. With regard to descriptions of $\mathrm{I}_{2} \mathrm{KI}$ solutions, researchers have used expressions based on total solute weight per volume or percentage of $\mathrm{I}_{2}$ alone; in some instances, no solution details are provided. In the present study, the description of the concentrations of the $\mathrm{I}_{2} \mathrm{KI}$ solutions was simplified in terms of percentages of $I_{2}$.

The optimized imaging settings established in the present study included a lower tube voltage and exposure time and a higher current than settings used in several previous micro-CT studies ${ }^{2,22,24}$ of excised hearts. The setting differences may reflect differences in the physical size of the samples, capabilities of the individual scanners, or imaging preferences of the investigators. The type of micro-CT scanner and imaging settings used are important determinants of image quality.

The main limitations of the present study were the small number of hearts assessed and the limited range of imaging settings evaluated. Although the settings used were partly limited by the technical ability of the scanner, the unit used was a commercially available micro-CT scanner with a wide range of scanning options. Evaluations performed at more time points within the first 48 hours could have also provided a more detailed examination of the uptake of iodine. Tissue distortion was not assessed in the present study but could be evaluated in future investigations through serial measurements of an axis of the heart or heart volume.

On the basis of the results of the present study, a micro-CT protocol with optimal sample preparation conditions in $\mathrm{I}_{2} \mathrm{KI}$ solution and optimal imaging settings that allowed evaluation of complex cardiac structures of excised hearts from healthy adult cats 
was established. These experimental methods could serve as a basis for further micro-CT studies of cardiac morphology of cats and development of optimal micro-CT protocols for other types of excised organs.

\section{Acknowledgments}

Funded by a National Institute for Health Research (NIHR) Clinical Doctoral Research Fellowship (Simcock; grant reference ICA-CDRF-2017-03-053); NIHR Career Development Fellowship (Arthurs; grant reference NIHR-CDF-2017-10-037); Fellowship and Medical Research Council Clinical Research Training Fellowship (Shelmerdine; grant reference MR/R00218/1 [jointly funded by the Royal College of Radiologists]).

This work was supported by the NIHR GOSH BRC. The views expressed are those of the authors and not necessarily those of the NHS, the NIHR or the Department of Health.

The authors declare that they have no competing interests.

\section{Footnotes}

a. Med X Micro-CT scanner, Nikon, Tring, England.

b. Parafilm, M Bemis, Oshkosh, Wis.

c. XLSTAT, version 2019.3.1, Addinsoft, Paris, France.

\section{References}

1. Gregg CL, Butcher JT. Quantitative in vivo imaging of embryonic development: opportunities and challenges. Differentiation 2012;84:149-162.

2. Aslanidi OV, Nikolaidou T, Zhao J, et al. Application of microcomputed tomography with iodine staining to cardiac imaging, segmentation, and computational model development. IEEE Trans Med Imaging 2013;32:8-17.

3. Eloot L, Buls N, Covens P, et al. Quality control of microcomputed tomography systems. Radiat Prot Dosimetry 2010;139:463-467.

4. Thiboutot J, Yuan W, Park HC, et al. Current Advances in COPD imaging. Acad Radiol 2019;26:335-343.

5. Jacob RE, Carson JP. Automated measurement of heterogeneity in CT images of healthy and diseased rat lungs using variogram analysis of an octree decomposition. BMC Med Imaging 2014;14:1.

6. Li KZ, Gao Y, Zhang R, et al. The effect of a manual in strumentation technique on five types of premolar root canal geometry assessed by microcomputed tomography and three-dimensional reconstruction. BMC Med Imaging 2011;11:14

7. Chen KC, Arad A, Song ZM, et al. High-definition neural visualization of rodent brain using micro-CT scanning and nonlocal-means processing. BMC Med Imaging 2018;18:38.

8. Al Faraj A, Shaik AS, Alnafea M. Intrapulmonary administration of bone-marrow derived M1/M2 macrophages to enhance the resolution of LPS-induced lung inflammation: noninvasive monitoring using free-breathing MR and CT imaging protocols. BMC Med Imaging 2015;15:16.

9. De Rycke LM, Boone MN, Van Caelenberg AI, et al. Micro computed tomography of the head and dentition in cadavers of clinically normal rabbits. Am J Vet Res 2012;73:227232.

10. Rubio-Martínez LM, Cruz AM, Gordon K, et al. Structural characterization of subchondral bone in the distal aspect of third metacarpal bones from Thoroughbred racehorses via micro computed tomography. Am J Vet Res 2008;69:14131422.

11. Vlaminck L, Cnudde V, Pieters K, et al. Histologic and microcomputed tomographic evaluation of the osseointegration of a nonresorbable bone substitute in alveli of ponies after tooth extraction. Am J Vet Res 2008;69:604-610.

12. Metscher BD. MicroCT for developmental biology: a versatile tool for high-contrast 3D imaging at histological resolutions. Dev Dyn 2009;238:632-640.

13. Kim JS, Min J, Recknagel AK, et al. Quantitative threedimensional analysis of embryonic chick morphogenesis via microcomputed tomography. Anat Rec (Hoboken) 2011;294:1-10.

14. Degenhardt K, Wright AC, Horng D, et al. Rapid 3D phenotyping of cardiovascular development in mouse embryos by micro-CT with iodine staining. Circ Cardiovasc Imaging 2010;3:314-322.

15. Gignac PM, Kley NJ. Iodine-enhanced micro-CT imaging: methodological refinements for the study of the soft-tissue anatomy of post-embryonic vertebrates. J Exp Zool B Mol Dev Evol 2014;322:166-176.

16. Pandit $\mathrm{P}$, Johnston SM, Qi Y, et al. The utility of micro-CT and MRI in the assessment of longitudinal growth of liver metastases in a preclinical model of colon carcinoma. Acad Radiol 2013;20:430-439.

17. Gufler H, Franke FE, Wagner S, et al. Fine structure of breast tissue on micro computed tomography a feasibility study. Acad Radiol 2011;18:230-234.

18. Sheikhzadeh M, Vercnocke AJ, Tao S, et al. Impact of effective detector pixel and ct voxel size on accurate estimation of blood volume in opacified microvasculature. Acad Radiol 2019;26:1410-1416

19. Payne JR, Brodbelt DC, Luis Fuentes V. Cardiomyopathy prevalence in 780 apparently healthy cats in rehoming centres (the CatScan study). J Vet Cardiol 2015;17(suppl 1):S244S257.

20. Peterson EN, Moise NS, Brown CA, et al. Heterogeneity of hypertrophy in feline hypertrophic heart disease. J Vet Intern Med 1993; 7:183-189.

21. Fox PR. Hypertrophic cardiomyopathy. Clinical and pathologic correlates. J Vet Cardiol 2003;5:39-45.

22. Hutchinson JC, Arthurs OJ, Ashworth MT, et al. Clinical utility of postmortem microcomputed tomography of the fetal heart: diagnostic imaging vs macroscopic dissection. Ultrasound Obstet Gynecol 2016;47:58-64.

23. Kim AJ, Francis R, Liu X, et al. Microcomputed tomography provides high accuracy congenital heart disease diagnosis in neonatal and fetal mice (Erratum published in Circ Cardiovasc Imaging 2013;6:e30). Circ Cardiovasc Imaging 2013;6:551-559.

24. Stephenson RS, Boyett MR, Hart G, et al. Contrast enhanced micro-computed tomography resolves the 3 -dimensional morphology of the cardiac conduction system in mammalian hearts. PLOS One 2012;7:e35299.

25. Pauwels E, Van Loo D, Cornillie P, et al. An exploratory study of contrast agents for soft tissue visualization by means of high resolution X-ray computed tomography imaging. $J \mathbf{M i}$ crosc 2013;250:21-31.

26. Dunmore-Buyze PJ, Tate E, Xiang FL, et al. Three-dimensional imaging of the mouse heart and vasculature using microCT and whole-body perfusion of iodine or phosphotungstic acid. Contrast Media Mol Imaging 2014;9:383-390.

27. Dullin C, Ufartes R, Larsson E, et al. muCT of ex-vivo stained mouse hearts and embryos enables a precise match between $3 \mathrm{D}$ virtual histology, classical histology and immunochemistry. PLoS One 2017;12:e170597.

28. Walton LA, Bradley RS, Withers PJ, et al. Morphological characterisation of unstained and intact tissue micro-architecture by $\mathrm{x}$-ray computed micro- and nano-tomography. Sci Rep 2015;5:10074

29. Hopkins TM, Heilman AM, Liggett JA, et al. Combining micro-computed tomography with histology to analyze biomedical implants for peripheral nerve repair. $J$ Neurosci Methods 2015;255:122-130.

30. Lombardi CM, Zambelli V, Botta G, et al. Postmortem microcomputed tomography (micro-CT) of small fetuses and hearts. Ultrasound Obstet Gynecol 2014;44:600-609.

31. Liu X, Tobita K, Francis RJ, et al. Imaging techniques for visualizing and phenotyping congenital heart defects in murine models. Birth Defects Res C Embryo Today 2013;99:93-105.

32. Pelizzo G, Calcaterra V, Lombardi C, et al. Fetal cardiac impairment in nitrofen-induced congenital diaphragmatic hernia: postmortem microcomputed tomography imaging study. Fetal Pediatr Pathol 2017;36:282-293. 
33. Aslanidi OV, Colman MA, Varela M, et al. Heterogeneous and anisotropic integrative model of pulmonary veins: computational study of arrhythmogenic substrate for atrial fibrillation. Interface Focus 2013;3:20120069.

34. Metscher BD. MicroCT for comparative morphology: simple staining methods allow high-contrast 3D imaging of diverse non-mineralized animal tissues. BMC Pbysiol 2009;9:11.

35. Cox PG, Jeffery N. Reviewing the morphology of the jaw- closing musculature in squirrels, rats, and guinea pigs with contrast-enhanced microCT. Anat Rec (Hoboken) 2011;294:915-928.

36. Gignac PM, Kley NJ, Clarke JA, et al. Diffusible iodinebased contrast-enhanced computed tomography (diceCT): an emerging tool for rapid, high-resolution, 3-D imaging of metazoan soft tissues. J Anat 2016;228:889-909.

37. Ritman EL. Micro-computed tomography-current status and developments. Annu Rev Biomed Eng 2004;6:185-208.

\section{Appendix}

Summary of reported conditions for preparation and micro-CT imaging of hearts from various species.

\begin{tabular}{|c|c|c|c|c|c|c|c|c|c|c|}
\hline Reference & $\begin{array}{c}\text { Status } \\
\text { of } \\
\text { heart }\end{array}$ & $\begin{array}{l}\text { Species } \\
\text { and } \\
\text { life stage }\end{array}$ & $\begin{array}{c}\text { Heart } \\
\text { weight } \\
\text { (g) }\end{array}$ & $\begin{array}{c}\text { No. } \\
\text { of } \\
\text { hearts }\end{array}$ & $\begin{array}{c}\text { Concentration } \\
\text { of } \mathrm{I}_{2} \mathrm{KI} \\
\text { solution }\end{array}$ & $\begin{array}{l}\text { Immersion } \\
\text { time (d) }\end{array}$ & $\begin{array}{c}\text { Details of } \\
\mathrm{I}_{2} \mathrm{KI} \text { solution } \\
\text { concentration } \\
\text { provided }\end{array}$ & $\begin{array}{c}\text { Tube } \\
\text { voltage (kV) }\end{array}$ & $\begin{array}{c}\text { Current } \\
(\mu \mathrm{A})\end{array}$ & $\begin{array}{l}\text { Exposure } \\
\text { time }(m s)\end{array}$ \\
\hline Aslanidi et a $\left.\right|^{2}$ & Excised & Adult Boxer & 256 & 1 & $7.5 \%$ & 7 & No & 150 & 125 & - \\
\hline Stephenson et $\mathrm{a}^{24}$ & Excised & Adult rat & $2.25-5.5$ & 4 & $1.87 \%-15.0 \%$ & 2 & No & $130-155$ & - & - \\
\hline Stephenson et $\mathrm{a}^{24}$ & Excised & Rabbit & 4.1 & 2 & $7.5 \%$ & 3 & No & $130-155$ & - & - \\
\hline Stephenson at a ${ }^{24}$ & Excised & Rabbit & 4.1 & 2 & $3.75 \%$ & 5 & No & $130-155$ & - & - \\
\hline Kim at al ${ }^{23}$ & In situ & Fetal mouse & - & 2,105 & $1.25 \%$ & $2-3$ & Yes & 80 & 100 & $500-800$ \\
\hline Hutchinson at al ${ }^{22}$ & Excised & Fetal human & I.I-5.3 & 6 & $63.25 \mathrm{mg} / \mathrm{mL}$ & 2 & Yes & $82-125$ & $50-135$ & $500-1,000$ \\
\hline Pelizzo et a $\left.\right|^{32}$ & In situ & Fetal rat & - & 29 & $5 \%$ & 90 & Yes & 80 & 300 & 300 and 1,050 \\
\hline
\end{tabular}

$-=$ Not specified. 